\title{
Arc Jet Facility Test Condition Predictions Using the ADSI Code.
}

\author{
Grant Palmer*, Dinesh Prabhu* \\ ERC Inc. at NASA Ames, Moffett Field, CA 94035 \\ Imelda Terrazas-Salinas ${ }^{\dagger}$ \\ NASA Ames Research Center, Moffett Field, CA 94035
}

The Aerothermal Design Space Interpolation (ADSI) tool is used to interpolate databases of previously computed computational fluid dynamic solutions for test articles in a NASA Ames arc jet facility. The arc jet databases are generated using an Navier-Stokes flow solver using previously determined best practices. The arc jet mass flow rates and arc currents used to discretize the database are chosen to span the operating conditions possible in the arc jet, and are based on previous arc jet experimental conditions where possible. The ADSI code is a database interpolation, manipulation, and examination tool that can be used to estimate the stagnation point pressure and heating rate for user-specified values of arc jet mass flow rate and arc current. The interpolation is performed in the other direction (predicting mass flow and current to achieve a desired stagnation point pressure and heating rate). ADSI is also used to generate 2-D response surfaces of stagnation point pressure and heating rate as a function of mass flow rate and arc current (or vice versa). Arc jet test data is used to assess the predictive capability of the ADSI code.

\section{Nomenclature}

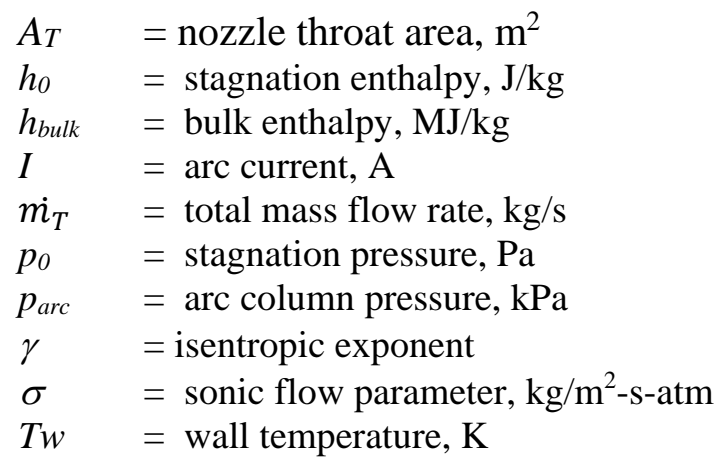

\section{Introduction}

Thermal protection system (TPS) materials used to protect spacecraft during planetary entry are typically tested and characterized in ground-based arc jet test facilities. The arc jet facilities available at NASA Ames Research Center include the Aerodynamic Heating Facility (AHF) [1]-[2], the Interaction Heating Facility (IHF) [2]-[4], the Panel Test Facility (PTF) [5], and more recently TP3 [6]. A schematic representing the IHF arc jet facility is shown in Figure 1. The arc jet plasma is generated when an electric arc forms between two electrodes separated by several hundred water-cooled copper arc heater segments [1]. The working gas, generally a mixture of air and argon, is heated to temperatures that can exceed $8000 \mathrm{~K}$. The flow then enters the plenum (section 2 in Fig. 1) where air is sometimes added to lower the enthalpy of the arc flow to meet a desired condition. The arc flow is then passed through a converging-diverging nozzle before exiting into the test chamber. A test article is placed downstream of the nozzle. By changing the nozzle shape and diameter, and by varying the gas mass flow rate and the arc current, a wide range of stagnation pressure and heat flux conditions can be achieved on the test article. 


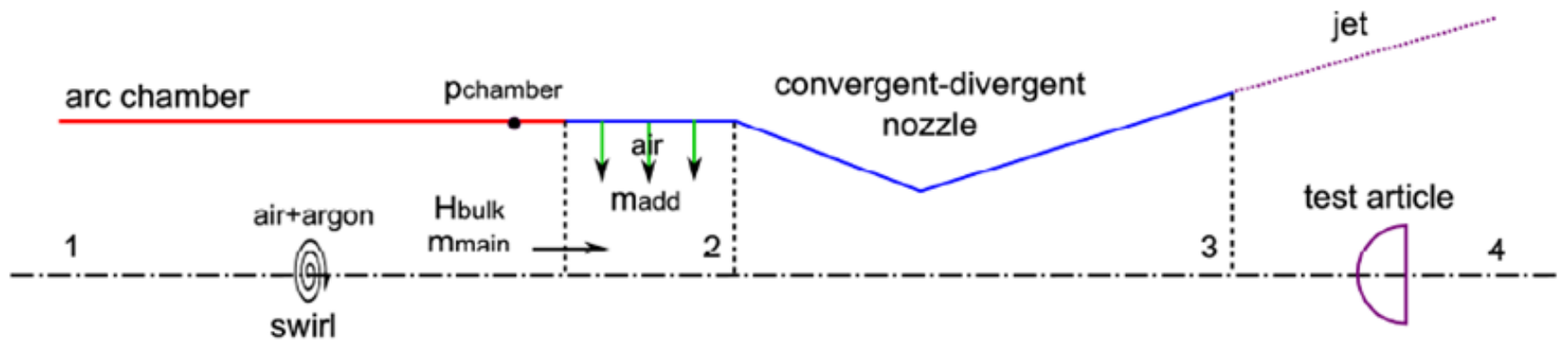

Fig. 1. Schematic of the NASA Ames IHF arc jet facility (taken from Ref. [4]).

A typical thermal protection material test mode in an arc jet is one in which target conditions, typically heat flux and pressure, are achieved at the stagnation point of test article (e.g. flat-faced cylinder, hemisphere, iso-q shape); the time of exposure to the arc-heated stream depends on the desired heat load or TPS material recession. The target conditions are usually cold-wall conditions, i.e., conditions that are achieved at the stagnation point of a calorimeter (copper slug or Gardon gauge) housed in a water-cooled copper body typically of the same shape and size as the test article. Flight vehicle assessments are generally at hot-wall boundary conditions, whereas arc jet test engineers typically design experiments based on cold-wall (i.e. water-cooled calorimeter) data. The process from going from experiment-to-flight assumes that: (i) a cold-wall to hot-wall heat rate conversion is available, or has been performed; and (ii) the effective hemispherical radius of the test article is known a priori. An example of an arc jet calorimeter, in this case a 4-inch diameter flat-faced cylinder, is shown in Fig. 2.

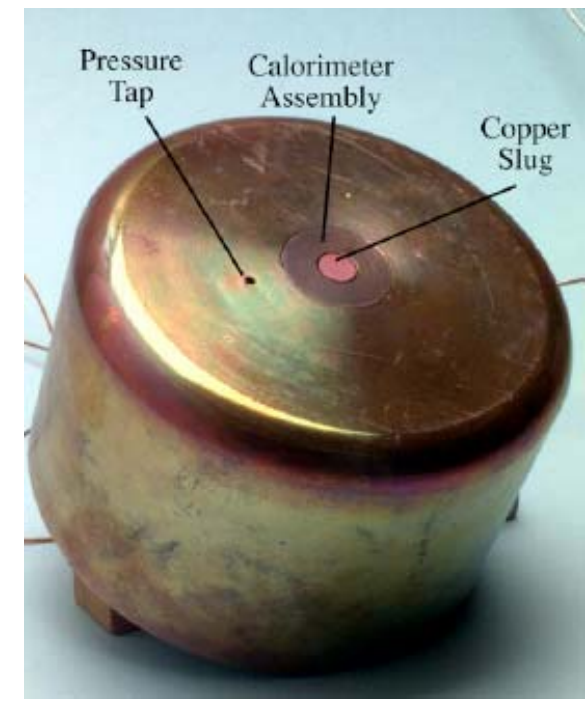

Fig. 2. 4-inch diameter flat-face cylinder calorimeter test article.

The heater settings - arc current and mass flow rate (including argon) - for the given target conditions are then determined from examining a database of prior tests. If the target conditions are within the test experience base, then small changes to the current and flow rates from prior tests of close proximity (in terms of current and flow rate) might be sufficient. However, if the target conditions lie outside the test experience, then an iterative process is necessary to determine the required arc-heater settings; the number of iterations depending on the level of accuracy levied on the target conditions. A CFD-based predictive capability that has been anchored to a large number of prior tests of calorimeters could be of use, considering the cost of testing and demand on the facility, in guiding arc-heater settings to achieve target conditions without the need for an active tuning process during testing.

In this paper, the Aerothermal Design Space Interpolation (ADSI) code [7] is used to interpolate previously computed databases of CFD arc jet facility solutions that were generated using the Data Parallel Line Relaxation 
$(D P L R)$ Navier-Stokes code [8]. The CFD databases consist of predicted stagnation point pressures and heating rates for a given geometry as a function of arc current, mass flow rate. The process for estimating CFD inflow conditions from arc current and mass flow rate are described in Section III. The CFD database used to test the ADSI code in this paper was for a 4" diameter hemisphere calorimeter in the AHF arc jet configured with an 18inch nozzle. The ADSI code is used to either estimate stagnation point pressure and heating rate for a given geometry based on a user-specified target point of arc current and mass flow rate, or it can be used to estimate the required arc current and mass flow rate to achieve a desired stagnation point pressure and heating rate. Arc jet tests typically involve trying to achieve a target heating rate, pressure, heat load, or maximum temperature on a given test article, and knowing the arc heater settings required to meet the target conditions can aid the arc jet test design process.

A series of tests were performed in the AHF arc jet facility at conditions that were not part of the CFD database. The primary objective of the test was to determine the efficacy and accuracy of the predictive method in determining arc-heater settings without the need for an active search during the operation of the facility. A secondary objective was to determine if the CFD-based predictive capability could lead to a decrease in measurement data scatter, and provide a firmer grasp on the relation between bulk enthalpy and arc-column pressure. The focus of this paper will be to assess whether interpolations of a previously computed arc jet CFD database can accurately reproduce and predict experimental data.

\section{CFD Grid Topology}

A sample arc jet grid topology, for the AHF 18-inch nozzle in this case, is shown in Fig. 3. The nozzle and model are symmetric, so the grids and CFD solutions are generated using an axisymmetric assumption. The nozzle is represented by the first grid zone (in pink).In Fig. 3, the nozzle domain includes the plenum. An alternative approach, discussed later in this paper, is to start the nozzle domain from the nozzle throat. The next three grid zones (green, cyan, and yellow) represent the free-jet section of the arc jet. An additional zone is sometimes included [3] outside of the green and cyan grid zones to more completely model the arc jet test box. Finally, the grid zone around the model itself (in black) must have enough grid resolution and shock alignment to accurately resolve surface quantities such as heating rate, pressure, and shear.

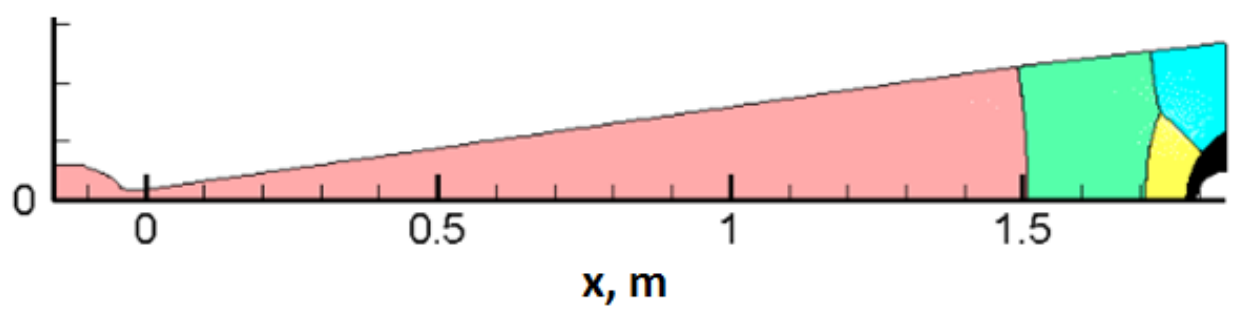

Fig. 3. Sample arc jet CFD grid topology, AHF facility with 18-inch nozzle.

\section{DPLR CFD Database}

The 64 CFD solutions in the AHF 4" hemisphere database were generated using the DPLR code, which has been previously used to simulate arc jet test flows [2]-[5]. A 6-species $\left[\mathrm{N}_{2}, \mathrm{O}_{2}, \mathrm{NO}, \mathrm{N}, \mathrm{O}, \mathrm{Ar}\right.$ ] finite-rate chemistry model was used for all solutions. The copper calorimeter wall was assumed to be fully catalytic (surface catalysis with constant catalytic efficiency factor, $\gamma=1$ ) with a cold-wall boundary condition with $T_{w}=400 \mathrm{~K}$.

The CFD database locations were defined in terms of mass flow rate and arc current, which are the quantities used by test engineers to specify arc jet experiment conditions. The CFD database used in this paper assumed no additional air was added in the plenum region (i.e. only main air and argon). The database solution locations are depicted in Fig. 4. A $0.1016 \mathrm{~m}$ (4-inch) diameter hemisphere calorimeter located $0.3048 \mathrm{~m}$ (12 inches) 
downstream of the nozzle exit has been previously tested 17 times in the AHF 18-inch nozzle under no add-air conditions. This previous experimental data is denoted by the red squares in Fig. 4. CFD solutions were run at these 17 conditions. An additional 47 CFD solutions, indicated by the black squares in Fig. 4, were run at values of arc current and mass flow rate that span the range of conditions achievable in the AHF. There are certain regions (i.e. high current/low mass flow and high mass flow/low current) that cannot be matched in the facility and were therefore excluded from the CFD databases. The blue squares in Fig. 4 indicate the conditions of the AHF-312 arc jet experiment that will be used to verify/validate ADSI.

One of the questions that will be addressed in this paper is whether the CFD database is needed at all. The ADSI code could be used to interpolate the existing experimental data to the desired test conditions. However, using a CFD database might result in better estimations of arc jet test conditions because (a) the existing experimental data may not span the entire operating environment of the facility and new target conditions may lie outside of the experimental data and (b) the increased density of the CFD database would reduce the interpolation error by reducing the size of the interpolation stencil.

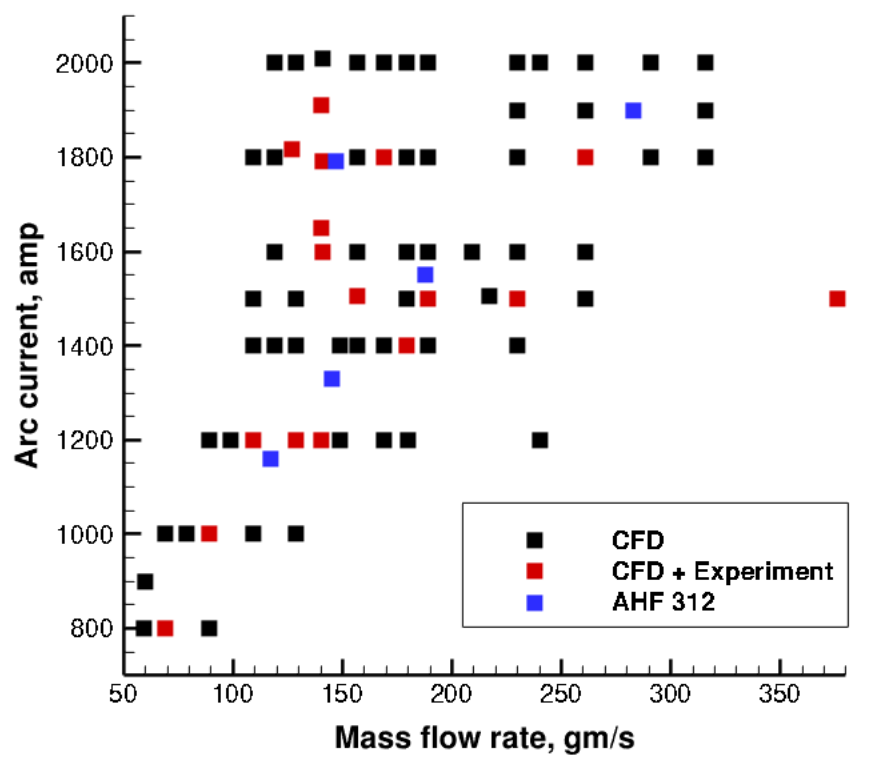

Fig. 4. AHF CFD database locations.

The accuracy of interpolating a CFD database using a tool such as ADSI depends on the accuracy of the underlying CFD solutions. One key to accurate arc jet CFD predictions is to properly specify the inflow boundary conditions at the nozzle throat or plenum. The arc jet test engineers specify the test conditions by setting the mass flow rates of air and argon and the arc current. This in turn sets the arc column pressure and bulk enthalpy of the flow. Generally speaking, the desired quantities to be measured on the test article include the stagnation point pressure and heating rate. As shown in Fig. 5, stagnation point pressure is approximately a linear function of arc column pressure. Stagnation point heating rate is a function of the bulk enthalpy. The commonly-used methods to estimate bulk enthalpy use correlations that are a function of mass flow rate and arc column pressure. 


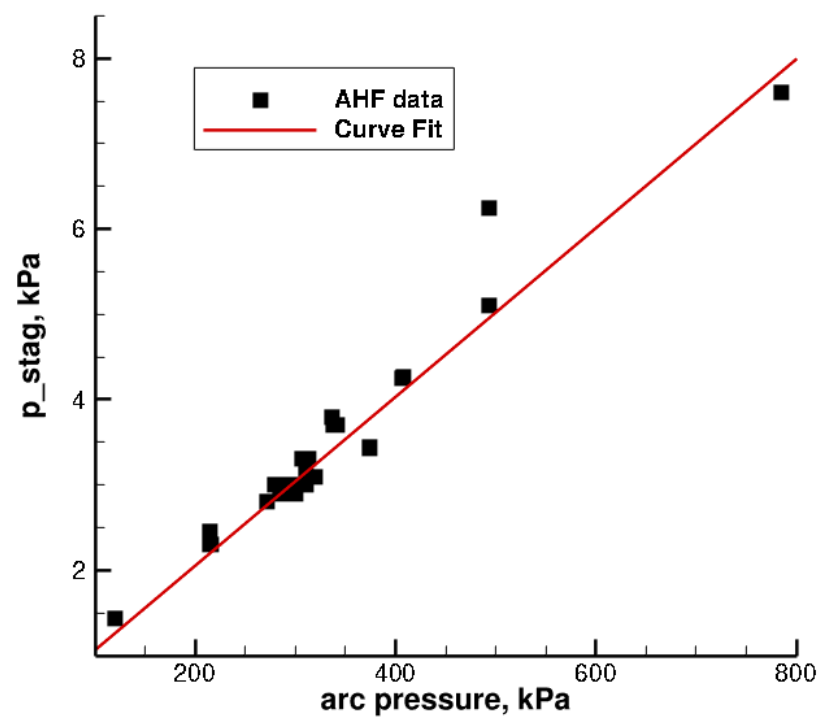

Fig. 5. Relationship of stagnation point pressure to arc column pressure, 4" diameter hemisphere model in the AHF arc jet with the 18” nozzle.

Arc column pressure is primarily a function of mass flow rate, with a lesser dependence on arc current. Arc column pressure is measured during an arc jet test, so post-test CFD can use the measured values. If pre-test CFD is desired, arc column pressure can be estimated using a 2-D response surface as a function of mass flow rate and current based on previously measured values. For the AHF arc jet with the 18-inch nozzle, a 2-D response surface was computed based on 36 known experimental arc column pressure measurements. The response surface equation, shown in Eq. (1), has a correlation coefficient of 0.9993 with the underlying experimental data.

$P_{\text {arc }}=62.967+2.0731 \dot{m}_{T}+0.0427 I+2.079 \times 10^{-4} \dot{m}_{T} I-5.748 \times 10^{-4} \dot{m}_{T} \dot{m}_{T}-1.387 \times 10^{-5} I^{2}$

The bulk enthalpy for a given arc jet test can be estimated using the Enthalpy By Energy Balance (EB2) approach by measuring the temperature rise of the water used to cool the arc jet [9]. For pre-test estimates of bulk enthalpy, several correlations have been developed over the years based on experimental EB2 measurements [10]. The bulk enthalpy correlation used in this paper was developed by Prabhu, et al [2] and estimates the bulk enthalpy as a function of the sonic flow parameter, $\sigma$, which is the ratio of the total mass flow rate to the arc column pressure and nozzle throat area. A graphical depiction of the Prabhu bulk enthalpy correlation and the experimental data it is based on can be seen in Fig. 6, which was taken from Ref. [2].

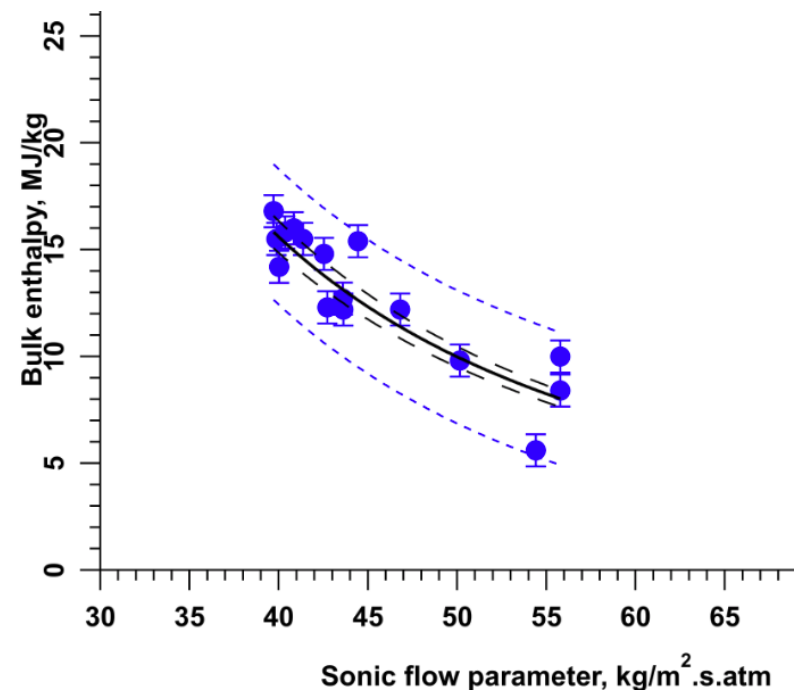


Fig. 6. Bulk enthalpy correlation used for arc jet inflow boundary condition (Ref. [2]).

The CFD inflow conditions can be determined once arc chamber pressure and bulk enthalpy are known. The AHF facility has sufficient mixing in the plenum such that a uniform inflow can be assumed. A single value of density, temperature, and velocity, and a single set of species mass fractions is used along the entire inflow line from the center of the inflow to the wall. If the CFD domain begins at the nozzle throat, the Mach number at the throat can be assumed to be sonic, and the pressure and enthalpy at the throat are assumed to follow the relations for 1-D isentropic flow. If the CFD domain starts at the plenum, the inflow velocity is assumed to be sufficiently small such that the inflow pressure and enthalpy are assumed to be equal to the arc column pressure and bulk enthalpy respectively. Full details of computing the inflow conditions for CFD arc jet simulations can be found in Ref. [2].

To assess the differences in selecting the nozzle throat or plenum as the inflow plane, CFD solutions were generated with both inflow types for a 4" hemisphere calorimeter at different levels of current and mass flow rate. The comparison of the surface heating rate profiles as a function of distance along the body from the stagnation point at two sets of conditions is shown in Fig. 7. In both of the cases tested, there was little difference in the surface solutions. The maximum difference in stagnation point heating rate was $1.1 \%$, which is an order of magnitude less than the uncertainty of experimental heating rate measurements. Therefore, either inflow condition could be used for the AHF simulations presented in this paper. CFD simulations for other arc jet facilities that are known to have non-uniformities in the inflow profiles, for example the NASA Ames IHF arc jet, may experience greater differences depending on the location of the CFD inflow boundary.
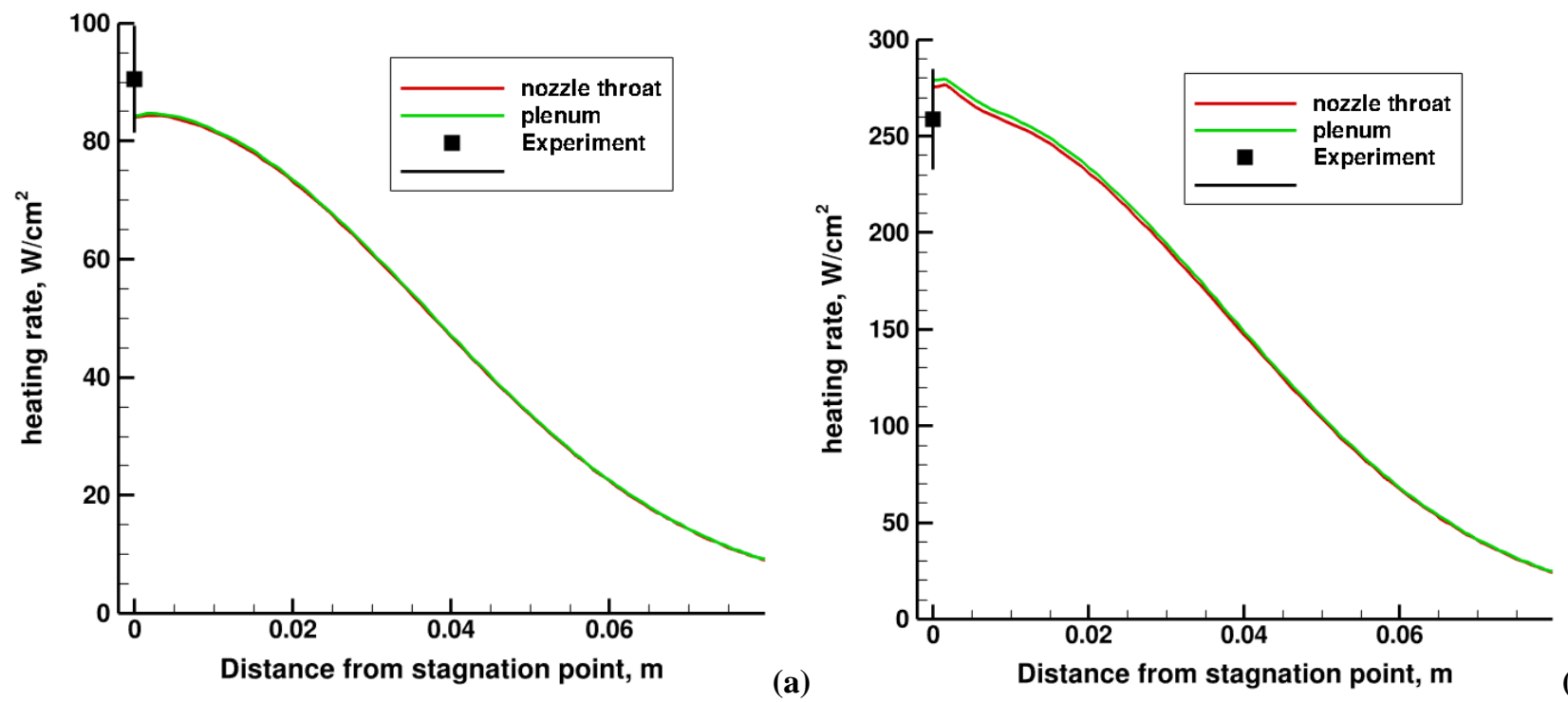

(b)

Fig. 7. Comparing surface heating rate profiles as a function of wetted distance from the stagnation point with nozzle throat and plenum inflows. (a) current $=800 \mathrm{~A}$, mass flow rate $=69 \mathrm{gm} / \mathrm{s}(\mathrm{b})$ current $=1500 \mathrm{~A}$, mass flow $=376 \mathrm{gm} / \mathrm{s}$.

Typically CFD is used to predict cold-wall conditions on a water-cooled copper calorimeter test article, so a standard CFD surface boundary condition is to assume a constant temperature, catalytic wall. Because the freestream enthalpy is much larger than the wall enthalpy, the heating rate is relatively insensitive to wall temperature. Values of 400 or $500 \mathrm{~K}$ for wall temperature are typically used.

There were 16 previous tests in the AHF with the 18-inch nozzle using the 4" hemisphere calorimeter with no additional air added in the plenum region. These conditions were included in the CFD database used in this work, and are locations where direct comparisons between the CFD and experimental stagnation point values can be made. The results of these comparisons are shown in Table 1. In some cases, there was more than one test at a given current and mass flow rate, so more than one experimental value is shown in the table. Typical uncertainties for AHF arc jet measurements are assumed to be $5 \%$ for pressure and $10 \%$ for heating rate [2]. The majority of 
the CFD results are within these experimental uncertainty levels. There were four cases that exceeded a 5\% difference in pressure, and in all cases the CFD heating rates were within $10 \%$ of the experimental value. There is some scatter to the experimental arc pressure values as seen in Fig. 5, and the error in the arc pressure response surface used to specify the CFD inflow conditions will vary somewhat at different values of mass flow rate and arc current. Generally speaking, the results in Table 1 indicate a reasonable confidence in the accuracy of the underlying CFD 4" hemisphere database used in this work.

Table 1. Comparison of CFD and experimental stagnation point values, 4" hemisphere model.

\begin{tabular}{|c|c|c|c|c|c|c|c|}
\hline \multirow{2}{*}{$\begin{array}{c}\text { Current, } \\
\text { Amp }\end{array}$} & Mass flow, & \multicolumn{3}{|c|}{ Stag point pressure, $\mathrm{kPa}$} & \multicolumn{3}{c|}{ Stag point heating rate, W/cm2 } \\
\cline { 3 - 8 } & gm/s & CFD & Experiment & \% diff & CFD & Experiment & $\%$ diff \\
\hline 800 & 69 & 1.404 & 1.43 & 1.8 & 84.4 & 90.5 & 6.8 \\
\hline 1000 & 79 & 1.596 & - & - & 92.3 & 89.1 & 3.6 \\
\hline 1200 & 99 & 2.063 & - & - & 119.4 & $129.8-131.8$ & 8.7 \\
\hline 1200 & 109 & 2.317 & $2.3-2.45$ & 2.5 & 134.3 & $126.5-130.4$ & 4.6 \\
\hline 1200 & 129 & 2.706 & - & - & 145.1 & $151-153$ & 4.6 \\
\hline 1200 & 140 & 2.972 & 3.0 & 1.0 & - & - & - \\
\hline 1400 & 179 & 3.737 & $3.43-3.44$ & 8.7 & 190.8 & $197.5-208.3$ & 5.9 \\
\hline 1505 & 157 & 3.651 & $3.7-3.8$ & 2.6 & 200.4 & $202-204$ & 1.3 \\
\hline 1500 & 189 & 4.316 & $4.25-4.26$ & 1.4 & 217.9 & $231.9-234.6$ & 6.6 \\
\hline 1500 & 230 & 5.138 & $5.08-5.11$ & 0.9 & 233.9 & $255.7-256.1$ & 8.6 \\
\hline 1500 & 376 & 7.838 & 7.6 & 3.1 & 278.9 & 258.7 & 7.8 \\
\hline 1600 & 141 & 3.186 & 3.1 & 2.8 & 181.4 & 178 & 1.9 \\
\hline 1650 & 140 & 3.181 & 3.2 & 0.6 & 182.9 & $181-184$ & 0.3 \\
\hline 1816 & 127 & 3.056 & 3.0 & 1.9 & 191.9 & 187 & 2.6 \\
\hline 1790 & 141 & 3.259 & 3.1 & 5.1 & 191.2 & 174 & 9.9 \\
\hline 1910 & 140 & 3.277 & 3.1 & 5.7 & 195.9 & $178-182$ & 8.8 \\
\hline
\end{tabular}

\section{ADSI Code}

The ADSI code is a database interpolation, manipulation, and examination tool that is written in the Java programming language. ADSI is typically run through a graphical user interface (GUI) but can also be run in batch-mode from the command line. The ADSI code has been extensively used in the Multi-Purpose Crew Vehicle (MPCV) Orion project [14] to interpolate aerodynamic and aerothermal loads database and for extracting data from the Smooth OML aerothermodynamic CFD database.

The ADSI GUI consists of a series of panels, each with a specific purpose (e.g. reading in databases and/or trajectories, data conversion, solution interpolation, etc.). A specialized "Arc Jet" panel was developed for the arc jet database interpolation. A screen shot of the ADSI "Arc Jet" panel is shown in Fig. 8. On the left-hand side of the window are the controls to specify the facility and test article inputs. Once these are specified, the corresponding CFD database is loaded into ADSI. More than one database can be loaded during a single run. A graphical depiction of the current active database is provided in the bottom right-hand corner of the GUI.

The ADSI code can interpolate the arc jet CFD database to a user-specified target point. The interpolation variables (e.g. mass flow and current or stagnation point pressure and heating rate) are chosen along with the target point values. Three interpolation schemes are currently implemented - a nearest neighbor approach based on a K-D tree, a linear scheme based on a Delaunay discretization of the database, and a simple inverse-distance method. When the target point and interpolation variables are selected, the interpolation is performed and the results appear in the lower left-hand corner of the GUI. The results can be saved to a file if desired. In addition to interpolations, ADSI can generate response surfaces for desired target values. These response surfaces provide derivatives of output variables as a function of input variables, for example rate of change of stagnation point 
heating rate as a function of arc current and mass flow, and can be used by test engineers to modify mass flow rate and arc current settings to achieve desired stagnation point values.

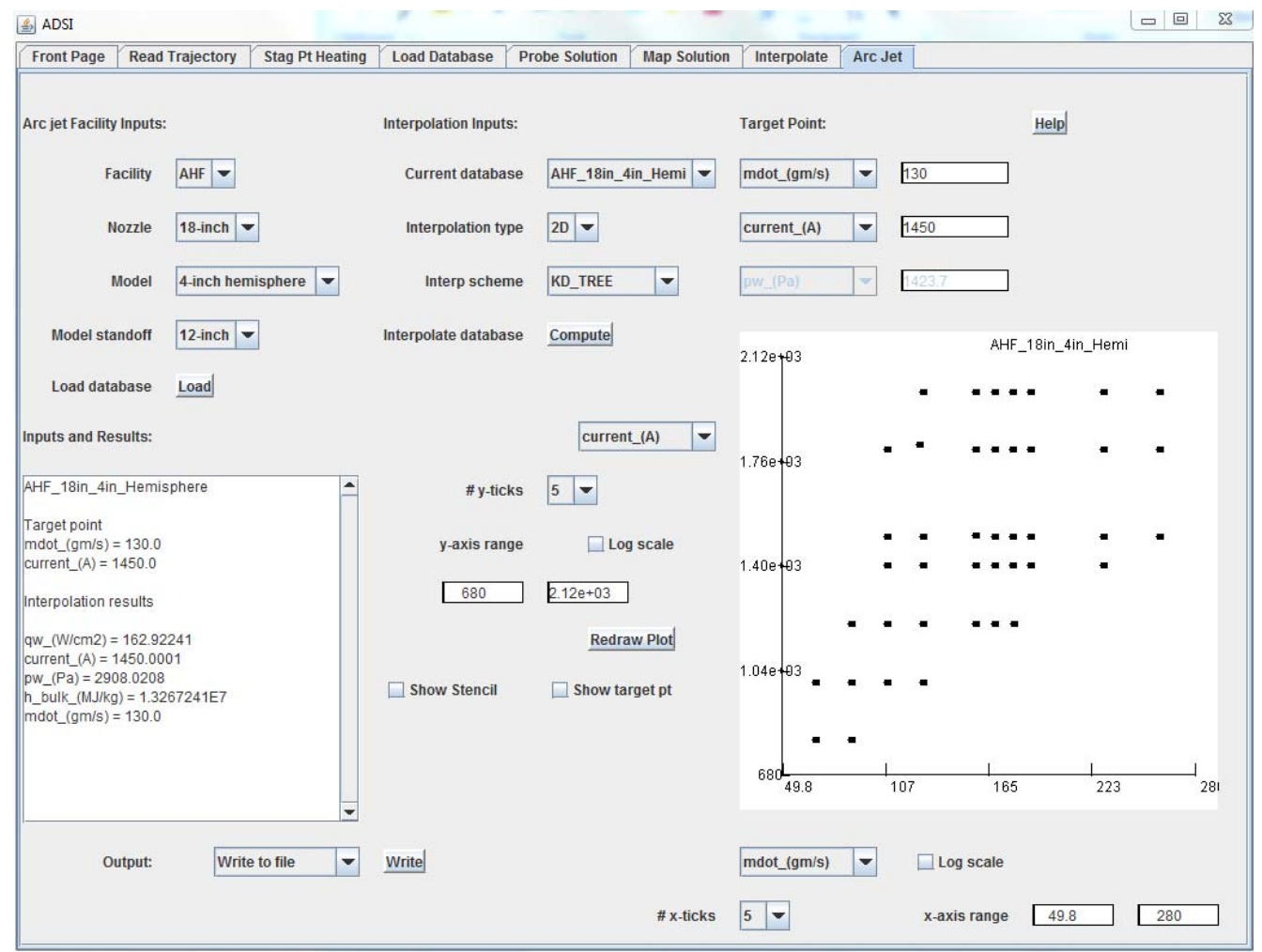

Fig. 8. ADSI arc jet panel screen shot.

\section{Validation/Verification of the ADSI Interpolation Approach}

An experiment was run in the AHF arc jet with the 18-inch nozzle (test designation AHF-312). Five 4" diameter hemisphere calorimeters were tested at various levels of arc current and mass flow rate. The test conditions were selected so they would not correspond to any test conditions in the current ADSI arc jet CFD database. The ADSI code was used to interpolate the 4" hemisphere database to the test conditions, and the ADSI stagnation point pressures and heating rates were compared to the experimental data. The ADSI code was also used to "reverse engineer" the test, meaning that ADSI was used to predict the arc current and mass flow rates required to meet the experimentally measured values of stagnation point pressure and heating rate.

The first test of ADSI was to use mass flow rate and arc current as the interpolation variables in an attempt to reproduce the stagnation point pressure and heating rate values measured during AHF-312. The ADSI values were computed using the K-D tree interpolation scheme. The comparison results are shown in Table 2. Also shown in the table are the arc current and mass flow rates used for each test condition. In some cases there were multiple measurements taken for a given set of test conditions, which is why there is more than one experimental value at some conditions.

The ADSI code was used to interpolate the CFD database and was also used to interpolate the previous experimental data for the 4-inch hemisphere calorimeter tested in the AHF 18-inch nozzle. The results for interpolated stagnation point pressure values are shown in Table 2. The first three columns contain the current and mass flow conditions for each test and the measured values of stagnation pressures. The ADSI values interpolated from the CFD database and the percent difference from the experimental data are shown in columns four and five. 
The interpolations from previous experimental data to the AHF-312 conditions and the percent differences are shown in columns six and seven.

The ADSI code was close to the expected 5\% uncertainty in stagnation point pressure for all five cases (slightly higher for two). The ADSI values tended to over-predict pressure. When interpolations are performed using the previous experimental data, the results are mixed. At two of the conditions, I=1550 and $1790 \mathrm{~A}$, the interpolated experimental data was within 5\% of the AHF-312 results. As seen in Fig. 4, these AHF-312 conditions were close to existing experimental data. The worst comparisons using the existing experimental data occurred at $\mathrm{I}=1330$ and 1900 A, which were not close to any existing experimental data. In fact, the AHF-312 condition where I=1900 Amp is outside of the existing experimental database, so an extrapolation is being performed. Generally speaking, using the CFD database to interpolate stagnation point pressure provides values closer to the actual AHF-312 data than are obtained using the existing experimental data.

Table 2. Interpolating stagnation point pressure based on arc current and mass flow rate.

\begin{tabular}{|c|c|c|c|c|c|c|}
\hline $\begin{array}{c}\text { Current, } \\
\text { Amp }\end{array}$ & $\begin{array}{c}\text { Mass flow, } \\
\text { gm/s }\end{array}$ & $\begin{array}{c}\text { AHF-312 } \\
\text { pressure, kPa }\end{array}$ & ADSI & \% diff & $\begin{array}{c}\text { Previous Expt } \\
\text { data only }\end{array}$ & \% diff \\
\hline 1160 & 117 & 2.3 & 2.415 & 5.0 & 2.522 & 9.7 \\
\hline 1330 & 145 & 2.9 & 3.067 & 5.7 & 3.274 & 12.8 \\
\hline 1550 & 188 & $4.1-4.2$ & 4.196 & 1.1 & 4.204 & 1.3 \\
\hline 1790 & 147 & 3.1 & 3.281 & 5.8 & 3.238 & 4.5 \\
\hline 1900 & 283 & 6.5 & 6.492 & 0.1 & 5.637 & 13.3 \\
\hline
\end{tabular}

Comparisons of interpolated values of stagnation point heating rate are shown in Table 3. When ADSI was used to interpolate the CFD database, the resulting heating rates were within the $10 \%$ uncertainty level for all five cases. The ADSI CFD interpolations tended to over-predict heating rate. When previous experimental data was interpolated to the AHF-312 conditions, the interpolated values matched reasonably well with the AHF-312 measurements for conditions where there was existing experimental data nearby, but large differences were seen for the I=1330 and 1900 Amp conditions where there was no existing experimental data close to the AHF-312 conditions.

The largest discrepancy between the ADSI/CFD interpolation and the AHF-312 data was 9.3\% at the I=1900 A condition. There are several possible reasons for the larger difference in heating rate at this condition. Looking at Fig. 4, the I=1900 A condition is in the upper right corner of the CFD database, in a region where the database cases are relatively sparse. The ADSI interpolation stencil for the I=1900 A case will cover a wider area and therefore be subject to greater interpolation error. The arc column pressure estimated from the response surface equation shows a relatively large error of 5.5\% from the value measured during the experiment at the I=1900 A conditions. Since the estimated value of bulk enthalpy is a function of the square of arc column pressure, a 5.5\% difference in arc column pressure corresponds to a $10.7 \%$ difference in bulk enthalpy.

Table 3. Interpolating stagnation point heating rate based on arc current and mass flow rate.

\begin{tabular}{|c|c|c|c|c|c|c|}
\hline $\begin{array}{c}\text { Current, } \\
\text { Amp }\end{array}$ & $\begin{array}{c}\text { Mass flow, } \\
\text { gm/s }\end{array}$ & $\begin{array}{c}\text { AHF-312 } \\
\text { heating, W/cm }\end{array}$ & ADSI & \% diff & $\begin{array}{c}\text { Previous Expt } \\
\text { data only }\end{array}$ & \% diff \\
\hline 1160 & 117 & 136 & 131.5 & 3.3 & 140.3 & 3.2 \\
\hline 1330 & 145 & 154 & 159.2 & 3.4 & 175.9 & 14.2 \\
\hline 1550 & 188 & $190-199$ & 208.1 & 7.0 & 215.1 & 10.6 \\
\hline 1790 & 147 & 174,178 & 181.2 & 3.0 & 180.7 & 2.7 \\
\hline 1900 & 283 & 249,254 & 275.0 & 9.3 & 201.1 & 20.0 \\
\hline
\end{tabular}

One of the purposes of the ADSI code is to serve as a predictive tool to help guide arc jet experiments in that it can estimate the arc current and mass flow rate needed to reach a target stagnation point pressure and heating rate. 
In this scenario, the pressure and heating rate are the interpolation variables, and the CFD database is interpolated to provide current and mass flow rate. There is a problem with this objective in that the interpolation of arc current based on stagnation point pressure and heating rate is not unique. As seen Fig. 9a, there is an approximately linear relationship between mass flow rate and stagnation point pressure for the 4-inch hemisphere CFD database. Similarly, the square root of mass flow rate varies approximately linearly with stagnation point heating. However, as shown in Fig. 9b, there is no correlation between arc current and stagnation point pressure. In other words, a target value of stagnation point pressure can be achieved with any number of arc current values.
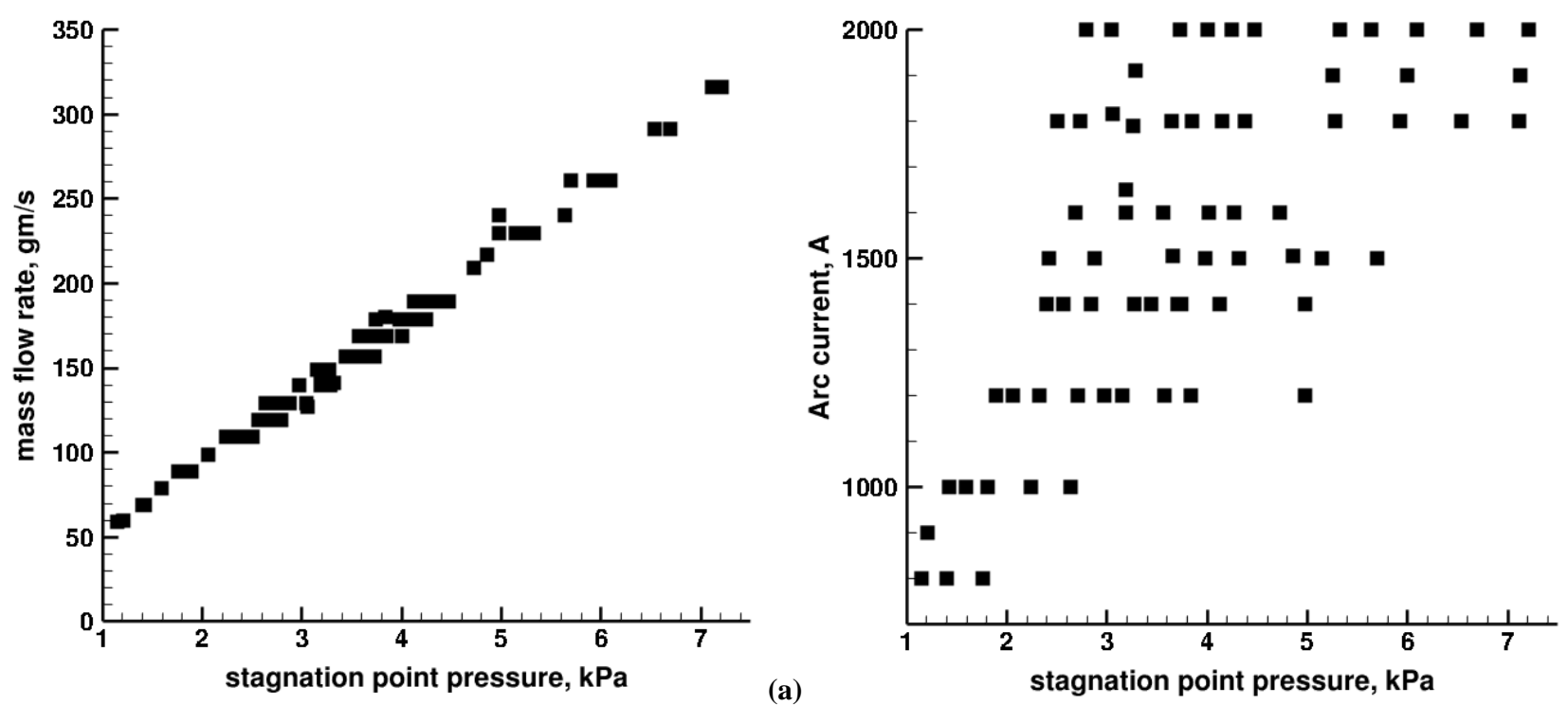

Fig. 9. Relationship of (a) mass flow rate and (b) arc current to stagnation point pressure.

The ADSI code was used to interpolate the CFD database and existing experimental data to estimate the mass flow rate necessary to achieve the AHF-312 values of stagnation point pressure and heating rate. The interpolated mass flow rate results for are shown in Table 4. The ADSI/CFD interpolated mass flow rates are within $10 \%$ of the AHF-312 values for all five cases and are within 5\% for three of them. If the existing experimental data is interpolated, the discrepancies are larger, exceeding $10 \%$ for two of the five cases. As was the case with the results in Tables 2 and 3, using the CFD database to interpolate improves the accuracy of the mass flow rate estimations.

Table 4. Interpolating mass flow rate based on stagnation point pressure and heating rate.

\begin{tabular}{|c|c|c|c|c|c|c|}
\hline $\begin{array}{c}\text { Pressure, } \\
\mathrm{kPa}\end{array}$ & $\begin{array}{c}\text { Heating } \\
\text { rate, W/cm2 }\end{array}$ & $\begin{array}{c}\text { AHF-312 mass } \\
\text { flow, gm/s }\end{array}$ & ADSI & \% diff & $\begin{array}{c}\text { Previous Expt } \\
\text { data only }\end{array}$ & \% diff \\
\hline 2.3 & 136 & 117 & 105.7 & 9.6 & 103.2 & 11.8 \\
\hline 2.9 & 154 & 145 & 131.3 & 9.4 & 133.5 & 7.9 \\
\hline 4.15 & 194.5 & 188 & 184.6 & 1.8 & 195.0 & 3.7 \\
\hline 3.1 & 176 & 147 & 139.8 & 4.9 & 141.0 & 4.1 \\
\hline 6.5 & 251.5 & 283 & 284.9 & 0.7 & 314.1 & 11.0 \\
\hline
\end{tabular}

One way to provide an initial estimate of arc current to achieve a target value of stagnation point pressure and heating rate is to first interpolate the CFD or experimental database to mass flow rate as was done in Table 4 and then use an inverse distance interpolation method to obtain the arc current based on the mass flow rate and stagnation point values. The results of this approach for the AHF-312 conditions are shown in Table 5. Estimating arc current by inverse distance interpolation of the CFD database produces values that are within $8 \%$ of the AHF312 settings for four of the five cases. Using the same approach with the existing experimental values results in larger differences except for the $\mathrm{I}=1790$ A condition where there was existing experimental data very close to the 
AHF-312 condition. The error using the existing experimental data was over $20 \%$ at the I=1900 A condition, where an extrapolation was necessary because the condition was outside of the experimental database.

Table 5. Estimating arc current based on mass flow rate and stagnation point conditions.

\begin{tabular}{|c|c|c|c|c|c|c|}
\hline $\begin{array}{c}\text { Pressure, } \\
\mathrm{kPa}\end{array}$ & $\begin{array}{c}\text { Heating } \\
\text { rate, W/cm2 }\end{array}$ & $\begin{array}{c}\text { AHF-312 arc } \\
\text { current, A }\end{array}$ & ADSI & \% diff & $\begin{array}{c}\text { Previous Expt } \\
\text { data only }\end{array}$ & \% diff \\
\hline 2.3 & 136 & 1160 & 1253 & 8.0 & 1279 & 10.2 \\
\hline 2.9 & 154 & 1330 & 1363 & 2.5 & 1478 & 11.1 \\
\hline 4.15 & 194.5 & 1550 & 1467 & 5.4 & 1712 & 10.5 \\
\hline 3.1 & 176 & 1790 & 1580 & 11.7 & 1695 & 5.3 \\
\hline 6.5 & 251.5 & 1900 & 1836 & 3.3 & 1503 & 20.9 \\
\hline
\end{tabular}

\section{Concluding Remarks}

In this paper, a CFD database of DPLR Navier-Stokes flow solutions was used to predict test conditions in the NASA Ames AHF arc jet facility. A 64-solution DPLR CFD database was generated for a 4" diameter hemisphere calorimeter based on values of arc current and mass flow rate that span the normal operating environment of the AHF facility. Based on previous experiments in the AHF arc jet, a CFD database can generally reproduce the experimental data to within $5 \%$ for stagnation point pressure and $10 \%$ for stagnation point heating rate. The ADSI code is used to interpolate the CFD database to estimate stagnation point pressure and heating rate based on input values of arc current and mass flow rate. The interpolation can also be performed in the other direction to predict the arc current and mass flow rate values required to reach a target value of stagnation point pressure and heating rate.

An arc jet experiment, designated as AHF-312, was performed in the AHF facility at conditions that were not part of the CFD database. The experimental data was used to validate/verify the approach of using the ADSI code to predict arc jet environments. Five different test conditions were run during the experiment. When ADSI was used to interpolate the CFD database to estimate stagnation point pressure based on input arc current and mass flow rate, the ADSI results were within $6 \%$ on pressure for all five test conditions. If the existing experimental data for the 4-inch hemisphere was used to estimate the AHF-312 stagnation pressures, the differences were larger, exceeding $10 \%$ for two cases where the AHF-312 conditions were not close to any existing experimental data. Similar results were seen when interpolating stagnation point heating rate. The ADSI/CFD interpolations were within $10 \%$ of the AHF-312 results for all five test conditions, and the interpolation of the existing experimental data resulted in differences larger than $10 \%$ for three of the conditions. Therefore, using the CFD database improved the predictions for stagnation point pressure and heating rates compared to using the existing experimental data for the AHF-312 test conditions.

Interpolations were also performed to estimate the required mass flow rates and arc current necessary to achieve target stagnation point heating rate and pressure values. This objective was complicated by the fact that the interpolation of arc current based on stagnation point values is not unique. To overcome this obstacle, mass flow rate was first interpolated based on stagnation point pressure and heating rate. Arc current was then estimated using an inverse distance scheme as a function of mass flow rate and stagnation point pressure and heating rate. Using this approach, ADSI was able to interpolate arc current from the CFD database to within 8\% of the AHF312 values for four of the five cases. Using the existing experimental data to estimate arc current resulted in larger discrepancies, exceeding $10 \%$ in four of the five cases. Based on the AHF-312 comparisons, it appears that using ADSI to interpolate a previously computed CFD database can improve the estimations of arc jet test conditions needed by the test engineers.

\section{Acknowledgements}

Support for Grant Palmer and Dinesh Prabhu was provided under NASA contract NNA10DE12C to ERC, Inc. 


\section{References}

[1] Stewart, D., Gokcen, T., and Chen, Y-K., "Characterization of Hypersonic Flows in the AHF and IHF NASA Ames Arc-Jet Facilities,” AIAA Paper 2009-4237, June 2009.

[2] Prabhu, D., Saunders, D., Oishi, T., Skokova, K., Santos, J., Fu, J., Terrazas-Salinas, I., Carballo, E., and Driver, D., "CFD Analysis Framework for Arc-Heated Flowfields, I: Stagnation Testing in Arc-jets at NASA ARC,” AIAA Paper 2009-4080, June 2009.

[3] Santos, J.A., Nawaz, A., Martinez, E., and Terrazas-Salinas, I., "Volumetric Heat Flux Characterization Experiments in the Interaction Heating Facility at NASA Ames,” AIAA Paper 2010-4785, June 2010.

[4] Winter, M.W., Raiche, G.A., Terrazas-Salinas, I., Hui, F.C.L., White, B., and Taunk, J.S., "Measurements of Radiation Heat Flux to a Probe Surface in the NASA Ames IHF Arc Jet Facility,” AIAA Paper 2012-3189, June 2012.

[5] Gokcen, T. and Alunni, A.I., "On Laminar to Turbulent Transition of Arc-Jet Flow in the NASA Ames Panel Test Facility,” AIAA Paper 2012-3304, June 2012.

[6] Peterson, K.H., Yount, B., Schneider, N.R., Prabhu,D.K., Arnold, J.O., Squire, T.H., Wercinski,P.F., ChavezGarcia, J.F., and Venkatapathy, E., "Thermal and Structural Performance of Woven Carbon Cloth for Adaptive Deployable Entry and Placement Technology, AIAA Paper 2013-1370, March 2013.

[7] Palmer, G.E., "Construction of CFD Solutions Using Interpolation Rather than Computation with the ADSI Code,” AIAA Paper 2009-0141, January 2009.

[8] Wright, M. W., White, T., and Mangini, N., "Data Parallel Line Relaxation (DPLR) Code User Manual Acadia - Version 4.01.1," NASA/TM-2009-215388, October 2009.

[9] Hightower, T. M., Balboni, J. A., MacDonald, C. L., Anderson, K. F., and Martinez, E. R., "Enthalpy by Energy Balance for Aerodynamic Heating Facility at NASA Ames Research Center Arc Jet Complex," $48^{\text {th }}$ International Instrumentation Symposium, The Instrumentation Systems, and Automation Society, Research Triangle Park, NC, May 2002.

[10] Thompson, C.S., Prabhu, D., Terrazas-Salinas, I., and Mach, J.J., "Bulk Enthalpy Calculations in the Arc Jet Facility at NASA ARC,” AIAA Paper 2011-3475.

[11] Winovich, W., "On the equilibrium sonic flow method for evaluating electric-arc air-heater performance,” NASA TN D-2132, March 1964.

[12] Shepard, C. E., Milos, F. S., and Taunk, J. S., “A sonic flow equation for electric arc jets,” AIAA Paper 1993-3183, July 1993.

[13] McBride, B.J. and Gordon, S., "Computer Program for Calculation of Complex Chemical Equilibrium Compositions and Applications. II. Users Manual and Program Description,” NASA RP-1311, 1996.

[14] Marshall, P. and Norris, S.D., “Orion Program Status,” AIAA Paper 2013-5476, June 2013. 